\title{
Anorectal Malignant Melanoma: Retrospective Analysis of Six Patients and Review of the Literature
}

\author{
Ibrahim Atak \\ Department of General Surgery, Ali Osman Sönmez Oncology Hospital, \\ Bursa, Turkey \\ Received July 28, 2018; Accepted October 5, 2018.
}

Key words: Malign melanoma - Rectum - Treatment - Laparoscopy

Abstract: Malignant melanomas are rare aggressive tumours originating from the pigment-producing melanocytes. In our study, a review of the literature and a retrospective analysis of patients undergoing surgery at our clinic due to anorectal malignant melanoma were performed. The information of 6 patients undergoing surgery in our clinic due to anorectal malignant melanoma between January 2010 and January 2018 was retrieved retrospectively. The patients were assessed regarding demographic data, physical examination and imaging findings, the surgical method performed, postoperative complication, histopathological findings, oncological treatment and follow-up results. Four of the patients were female and 2 were male and the mean age was $61.6(46-83)$ years. Two patients (33\%) had liver metastases at the time of initial presentation. Abdominoperineal resection (APR) was performed in all patients 3 with laparoscopic method. The mean length of hospital stay was recorded to be $6.5 \pm 1$ days (5-12 days). Adjuvant chemotherapy and radiotherapy were administered in all patients. Also, interferon treatment was administered in one patient additionally. During the follow-up, 4 patients died due to extensive metastatic disease determined approximately in the $13^{\text {th }}$ month. Two patients with regular follow-up are well and free of disease and their mean postoperative lifetime has been determined to be 12.5 months (6-26 months). Anorectal malignant melanomas (ARMM) are rare but aggressive tumours. The treatment should be focused on minimizing morbidity and maximizing the quality of life and function while removing the gross tumour.

Mailing Address: Dr. Ibrahim Atak, Department of General Surgery, Ali Osman Sönmez Oncology Hospital, Prof. Dr. Orhan Kamıș street No. 3, Osmangazi, Bursa, Turkey; Phone: +90 22422382 00; Fax: +90 22422510 51; e-mail:driatak@yahoo.com 


\section{Introduction}

Malignant melanomas are rarely seen aggressive tumours that arise from the pigment-producing melanocytes. The most common sites of malignant melanoma is the skin, followed by eyes and the anorectal region (Bolivar et al., 1982; Ceccopieri et al., 2000). Anorectal malignant melanomas (ARMM) have been described for the first time by Moore in 1987 and they account for $0.2-3 \%$ of all malignant melanomas and $0.1-4.6 \%$ of all rectal malignant tumours (Ojima et al., 1999; Damodaran et al., 2008). It is usually seen in the $5^{\text {th }}$ and $6^{\text {th }}$ decades in the adults and the females are affected much more than the males (Che et al., 2011). Usually, they nearly always cause similar symptoms of rectum cancer such as rectal bleeding, change in bowel habits, anal mass. Since ARMM is not sensitive to radiotherapy and chemotherapy, these treatment methods have a limited use. Surgical resection is the main therapeutic method for ARMM. Due to the delay in diagnosis and aggressive course of ARMM, approximately $26 \%$ of the patients have metastatic disease at the time of diagnosis. Therefore, the prognosis of ARMM is poor (Thibault et al., 1997). Since the incidence of the disease is low, studies related to ARMM are insufficient. Relevant information in the literature is usually as case presentation and case series. In this study, a review of the literature and a retrospective analysis of patients undergoing surgery at our clinic between 2010 and 2018 due to anorectal malignant melanoma were performed.

\section{Material and Methods}

The patients with anorectal malignant melanoma undergoing surgery at our Department of General Surgery between 2010 and 2018 were included in the study. The data of the patients were retrieved retrospectively and their demographic data, physical examination and imaging findings, the surgical method performed, the number of lymph nodes resected, histopathological findings, the length of hospital stay, morbidity and mortality, the presence of local recurrence or metastasis, follow-up results were assessed.

During the preoperative period, detailed histories of all patients were obtained, and their physical examinations were performed. Blood tests, posterior-anterior chest graph, serum carcino-embryonic antigen was routinely performed. Invasion to the surrounding tissues and distant metastasis of the tumour were assessed by using contrast-enhanced thoracoabdominal computed tomography (CT) and pelvic magnetic resonance imaging (MRI). Each patient diagnosed before surgery was referred to the relevant departments for an appropriate preoperative ophthalmologic and dermatological evaluation.

\section{Results}

Six patients have been operated due to anorectal malignant melanoma between January 2010 and January 2018. Four of patients (66.6\%) were females and 2 of 


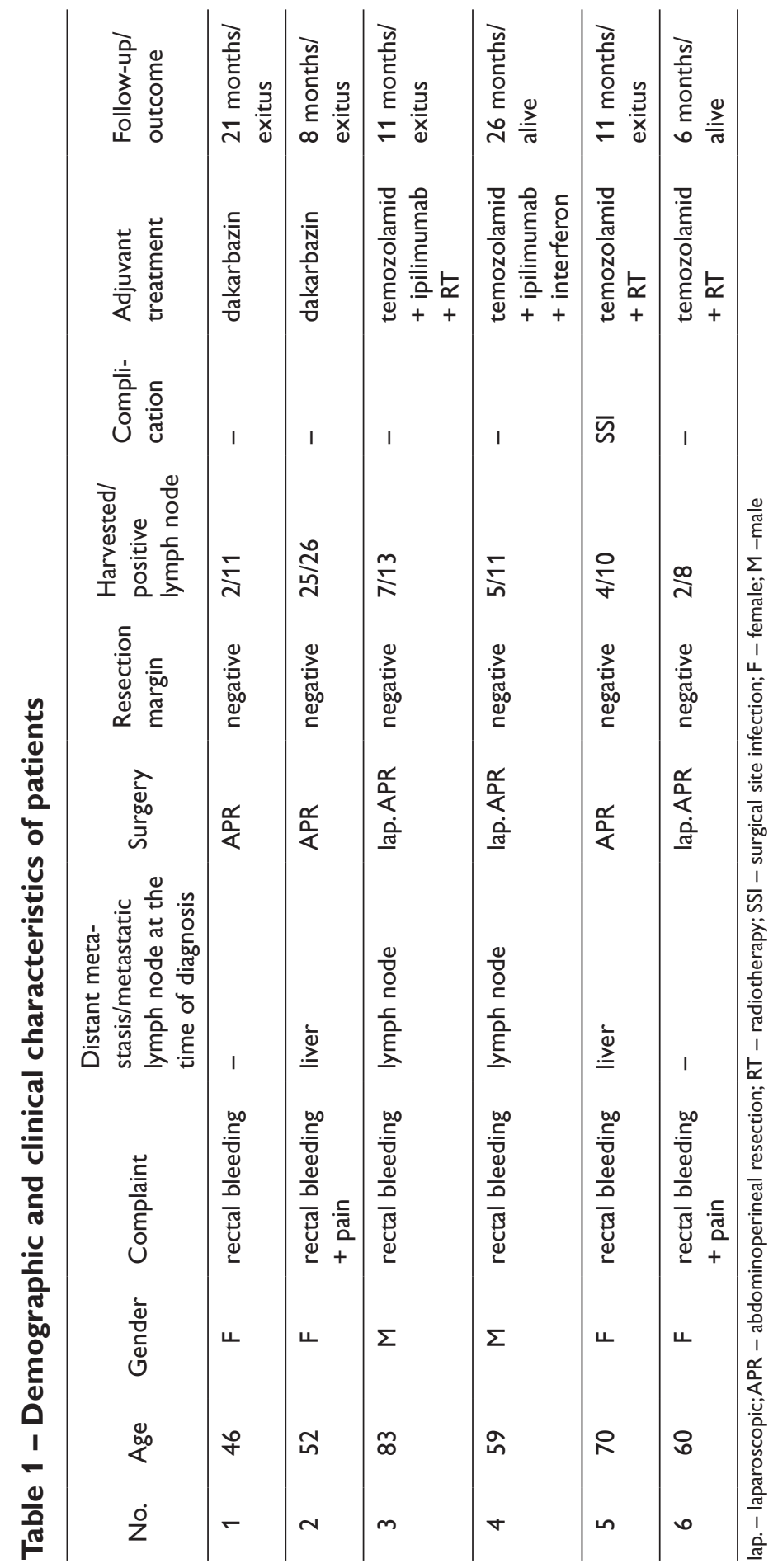


them (33.3\%) were males and the mean age was $61.6(46-83)$ years. All the patients had a complaint of rectal bleeding, 2 patients had a severe anal pain in addition to these findings (Table 1). The average length of the complaint was $10.75 \pm 3.9$ months (6-18 months). While no additional finding was determined at routine physical examination performed, an immobile, polypoid ulcerovegetative mass with ill-defined margins beginning from anoderm and extending to the lumen and partially narrowing of the lumen was determined in all of the patients in the rectal digital examination.

There was liver metastasis in 2 (33\%) of 6 patients during the initial presentation. Abdominoperineal resection (APR) was performed for palliation in 2 patients determined distant metastasis in the preoperative period due to the presence of both bleeding and obstruction findings and APR was performed in other patients for curative treatment in accordance with oncological principles. Three APR procedures were performed through a laparoscopic approach. No complication was determined except a postoperative surgical site infection occurred in one patient. The mean length of hospital stay was recorded to be $6.5 \pm 1$ days (5-12 days). Surgical margin was reported to be intact in all specimens at histopathological examination. The mean diameter of the masses was measured to be $5.5 \mathrm{~cm}(3-7 \mathrm{~cm})$ (Table 2).

A malignant tumour composed of normal colonic mucosa and afterwards tumour islands including usually fusiform cells was observed at histopathological examination (Figure 1). The tumour was composed of pleomorphic cells with large hyperchromatic nuclei, and large cytoplasm. A higher mitotic index was observed. Atypical mitoses were a striking feature. Neoplastic cells were observed to be positive for S-100 and HMB-45 and Melan A in the immunohistochemical examination (Figure 2). Adjuvant chemotherapy and radiotherapy were administered in all of the patients. Also, interferon treatment was administered in one patient additionally. During follow-up, 4 patients died due to extensive metastatic disease determined approximately in the $13^{\text {th }}$ month. Two patients with

\section{Table 2 - Histopathological features}

\begin{tabular}{ccccccccc}
\hline No. & $\begin{array}{c}\text { Tumour } \\
\text { diameter } \\
(\mathrm{cm})\end{array}$ & $\begin{array}{c}\text { Invasion } \\
\text { depth } \\
(\mathrm{mm})\end{array}$ & Grade & $\begin{array}{c}\text { Melan } \\
\text { A }\end{array}$ & $\begin{array}{c}\text { S-100 } \\
\text { protein }\end{array}$ & $\begin{array}{c}\text { Pan- } \\
\text { keratin }\end{array}$ & HMB-45 & $\begin{array}{c}\text { BRAF } \\
\text { mutation }\end{array}$ \\
\hline 1 & 3 & 2 & 2 & NA & + & - & NA & NA \\
2 & 7 & full thickness & 4 & NA & + & NA & + & NA \\
3 & 5 & 15 & 3 & - & + & - & + & - \\
4 & 6 & 3 & 2 & + & + & NA & + & - \\
5 & 7 & 3 & 4 & + & + & - & + & NA \\
6 & 5 & 17 & 3 & + & + & NA & + & - \\
\hline
\end{tabular}

NA - not recorded 


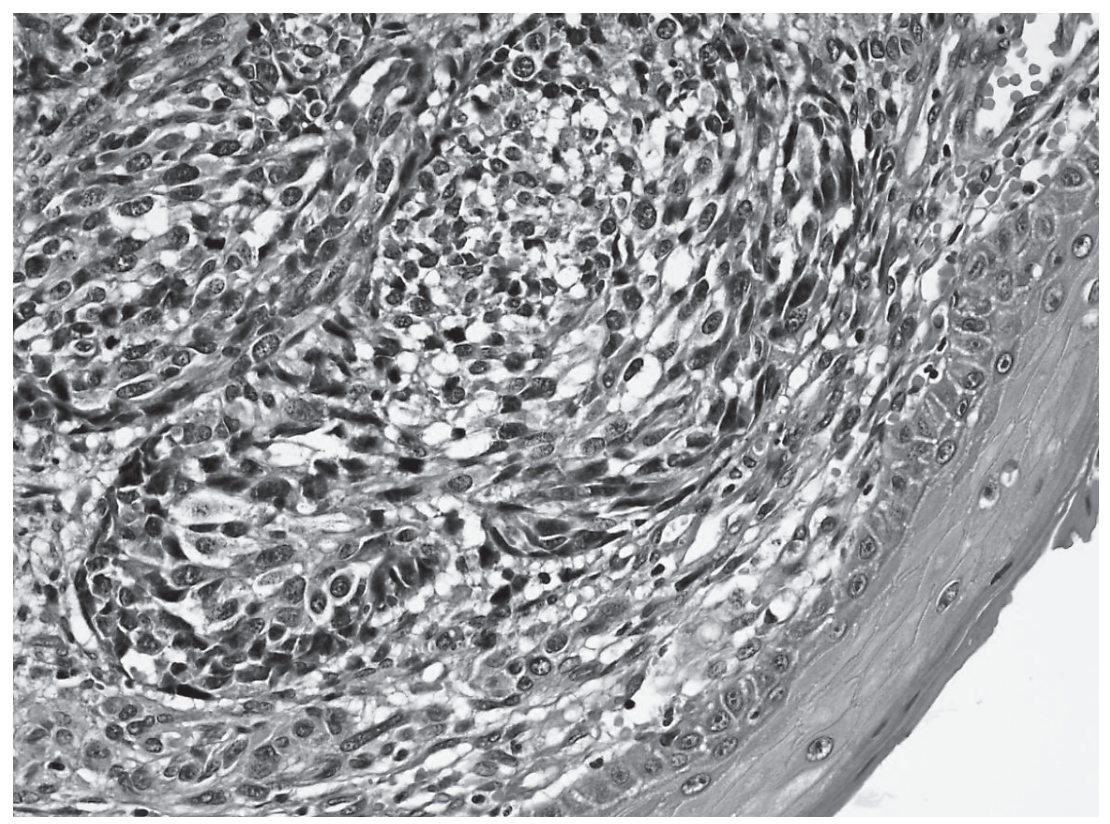

Figure 1 -Tumour observed in the subepitelial area is composed of epiteloid and fusiform-looking cells (hematoxylin-eosin, 40x).

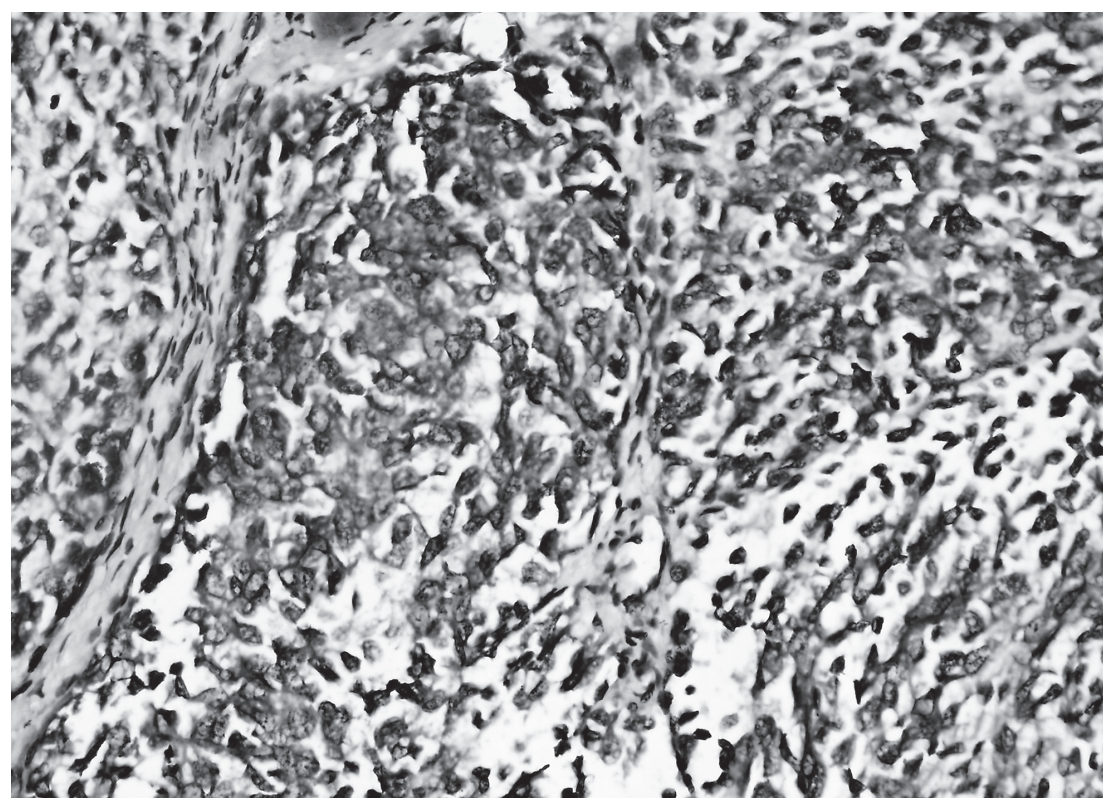

Figure 2 - HMB-45 positivity in neoplastic cells (hematoxylin-eosin, 40x). 
regular follow-up are well and free of disease and their mean postoperative lifetime has been determined to be 12.5 months (6-26 months).

\section{Discussion}

While ARMMs are quite rarely seen tumours, they are most frequently located in this region of the gastrointestinal tract. They can arise from melanocytes in the non-keratinized stratified squamous epithelium below the dentate line. Although anorectal malignant melanomas are seen at any age, they have been reported to be observed usually in 50-60 years of age and most commonly in females (54\% to $76 \%$ ). In keeping with the literature, females are higher also in our series and the majority of our patients is in this age group. Since ARMMs have a polypoid appearance macroscopically, they can be frequently mistaken for some benign conditions such as thrombosed hemorrhoids or rectal polyps (Ceccopieri et al., 2000). While the majority of patients with ARMM have pigmented melanomas, $16-53 \%$ of the cases have amelanotic melanomas (Sielezneff et al., 1993). There is no disease specific complaint and the most common symptom at presentation is rectal bleeding (54-78\%). Rectal mass (12-16\%), pain (14-27\%), obstipation (6\%) and diarrhoea (4\%) are among other symptoms (Wanebo et al., 1981; Goldman et al., 1990; Brady et al., 1995). The rectal digital examination provides information about ulceration, size, and fixation of the tumour for diagnostic evaluation. If melanin pigmentation is present clearly, rectosigmoidoscopy may support anorectal melanoma. Endorectal ultrasonography, CT, and MRI may provide valuable information in the evaluation of tumour size and presence of regional lymph node metastases. Histopathologically, ARMMs show considerable variability regarding the size and type of cells. They can mimic malignant lymphoma, small round cell sarcoma, spindle cell sarcoma, gastrointestinal stromal tumour and epidermoid carcinoma. Immunohistochemical (IHC) staining may be beneficial particularly in the patients with amelanotic melanoma where the making diagnosis is difficult. The most commonly used IHC stain in the diagnosis of ARMM is Anti-S-100 protein and it is highly sensitive for melanocytic differentiation (Chute et al., 2006). Also, HMB-45 and Melan A antibody are the stains specific for melanocytes used for diagnosis of malignant melanoma.

There are two methods used for staging of anorectal malignant melanoma. First one is the melanoma staging system of the American Joint Committee on Cancer (AJCC) and this system is based on the depth of primary tumour and tumour invasion to lymph nodes (Chang et al., 1998). In another staging system, staging is performed based on the depth of tumour invasion (stage 1,2), regional or lymphatic metastasis (stage 3) and distant metastasis (stage 4) (Falch et al., 2013). According to the AJCC melanoma staging, 2 of our patients were stage 2, 2 of them were stage 3 and 2 of them were stage 4 .

Treatment for ARMM is still controversial. Primer treatment modality is surgery. APR and wide local excision (WLE) are methods that can be chosen in the 
surgical treatment. It is suggested that APR can decrease the possibility of local recurrence by means of controlling spread to the mesenteric lymph nodes and obtaining a wider negative resection margin (Damodaran et al., 2008; Choi et al., 2011). However, since APR is associated with a higher morbidity and mortality and causes a poorer quality of life due to permanent colostomy, wide local excision is preferred by some surgeons (Pessaux et al., 2004). In their study including 85 patients, Brady et al. (1995) demonstrated that APR had a better 5-year survival rate compared to WLE ( $27 \%$ vs. $5 \%$ ) and they offered APR as a surgical method for ARMM treatment particularly in smaller tumours with no evidence of nodal metastases. Although in the study performed by Thibault et al. (1997) and including 37 patients, no significant difference was determined between WLE and APR regarding survival. Again, in the retrospective study performed by Yeh et al. (2006) comparing WLE and APR, no significant difference was determined between two groups regarding relapse and survival. Similar results were reported also by Yap and Neary (2004) in consequence of the investigation of 17 case series including 485 patients. In another recent study comparing WLE and APR, no significant difference was determined between the two groups regarding recurrence-free survival and disease-specific survival (Perez et al., 2013). Local recurrence after APR and WLE is reported to be $21-25 \%$ and $26-58 \%$; respectively (Pessaux et al., 2004; Yeh et al., 2006; Row and Weiser, 2009). Considering local recurrence, APR seems to be a more favourable method. Also, laparoscopic abdominoperineal resection developed for the first time by Ramalingam et al. in 2009 can be used for the treatment of ARMM and it has been reported that morbidity could be reduced with this method. An APR was performed in all of our patients except 3 patients undergoing laparoscopic APR and no major complication was determined except a postoperative surgical site infection.

Sentinel lymph node biopsy (SLNB) is a method caused a radical change in the treatment and prognosis of cutaneous melanoma and breast carcinoma. Since metastasis occurs to the inguinal and mesenteric lymph nodes in ARMM, SLNB is an applicable method (Tien et al., 2002). But the efficiency of the procedure is not known. SLNB can be performed in patients with ARMM in order to determine inguinal lymph nodes not detected clinically and then to perform curative resection and accurate staging of the tumour. However, studies conducted until now are insufficient to show meaningful results. When the nodal disease is identified, complete lymphadenectomy is frequently recommended, but a significant survival difference was not determined between the patients with and without a complete lymph node dissection. The role of performing a regional lymph node dissection is controversial in the surgical treatment of ARMM. Prophylactic inguinal lymph node dissection in ARMM could not be shown to improve survival (Reid et al., 2011; Stefanou and Nalamati, 2011).

There is no established standard adjuvant chemotherapy regimen in ARMM and particularly in metastatic disease. Some agents used in malignant cutaneous 
melanoma are considered to may have utility in the treatment. Although it may be stated that satisfactory results can be achieved with curative APR and adjuvant chemotherapy, there have been no randomized controlled studies performed specifically on this subject (Ishizone et al., 2008). However, administering chemotherapy alone without surgery has no benefit in the treatment (Yap and Neary, 2004). Although cytotoxic chemotherapeutic agents such as cisplatin, dacarbazine, vinblastine, vincristine, temozolomide, and interferon B, interleukin 2 are agents that can be used in adjuvant therapy, no benefit has been achieved in none of them regarding survival (Malik et al., 2002). Dacarbazine is the most frequently used agent and response to this medication is only about $20 \%$. Adjuvant radiotherapy after surgical excision is another method that can be used in the treatment of ARMM. It has been shown that radiotherapy after local excision decreased local recurrence from $50 \%$ to $17 \%$ compared to WLE alone (Kelly et al., 2011). No benefit of radiotherapy could be demonstrated other than decreasing local recurrence. Recently, use of immunomodulatory agents in cancer treatment is increasing. Immunotherapy supports anticancer immune response. Immunochemotherapy including systemic chemotherapeutic agents and immunomodulators (IL-2) has been shown to provide partial benefit in the treatment of ARMM in some studies (Ballo et al., 2002). In our series, dacarbazine, temozolomide + ipilimumab + RT, temozolomide + ipilimumab + interferon, and temozolomide + RT were administered in 2, 1, 1 and 2 patients; respectively.

Despite all treatment methods, the prognosis of the disease is significantly poorer compared to adenocarcinoma and cutaneous melanoma located in the same region. The 5 -year survival rate is less than 20\% (Weinstock, 1993; Brady et al., 1995; Chang et al., 1998). Survival of recurrent or metastatic disease is less than 10 months (Weinstock, 1993; Brady et al., 1995; Thibault et al., 1997). Survival is primarily dependent on locoregional lymph nodes and distant metastasis and it is irrespective of surgical treatment method performed. The depth of tumour infiltration, the presence of perineural invasion, age greater than 60 years and a tumour size of more than $1 \mathrm{~cm}$ in diameter are prognostic factors (Brady et al., 1995; Cagir et al., 1999; Yeh et al., 2006; Ishizone et al., 2008). In our series, 4 patients died due to the widespread metastatic disease at a mean of 13 months. Remaining 2 patients remain disease-free at postoperative $6^{\text {th }}$ and $26^{\text {th }}$ months.

In conclusion, ARMMs are rare but aggressive tumours. There is no specific symptom of the disease. It should be kept in mind particularly in the patients with rectal bleeding. All available possibilities should be used for preoperative staging. Since the tumour stage is among the most important prognostic factors, early diagnosis of the disease is of vital importance. It should be focused on minimizing morbidity and maximizing the quality of life and function while removing the gross tumour in the treatment. 


\section{References}

Ballo, M.T., Gershenwald, J. E., Zagars, G. K., Lee, J. E., Mansfield, P. F., Strom, E. A., Bedikian, A.Y., Kim, K. B., Papadopoulos, N. E., Prieto, V. G., Ross, M. I. (2002) Sphincter-sparing local excision and adjuvant radiation for anal-rectal melanoma. J. Clin. Oncol. 20, 4555-4558.

Bolivar, J. C., Harris, J.W., Branch, W., Sherman, T. S. (1982) Melanoma of the anorectal region. Surg. Gynecol. Obstet. 154, 337-341.

Brady, M. S., Kavolius, J. P., Quan, S. H. (1995) Anorectal melanoma. A 64-year experience at Memorial Sloan-Kettering Cancer Center. Dis. Colon Rectum 38, 146-151.

Cagir, B., Whiteford, M. H., Topham, A., Rakinic, J., Fry, R. D. (1999) Changing epidemiology of anorectal melanoma. Dis. Colon Rectum 42, 1203-1208.

Ceccopieri, B., Marcomin, A. R., Vitagline, F., Fragapane, P. (2000) Primary anorectal malignant melanoma: report of two cases. Tumori 86, 356-358.

Chang, A. E., Karnell, L. H., Menck, H. R. (1998) The National Cancer Data Base report on cutaneous and noncutaneous melanoma: a summary of 84,836 cases from the past decade. The American College of Surgeons Commission on Cancer and the American Cancer Society. Cancer 83, 1664-1678.

Che, X., Zhao, D. B., Wu, Y. K., Wang, C. F., Cai, J. Q., Shao, Y. F., Zhao, P. (2011) Anorectal malignant melanomas: retrospective experience with surgical management. World J. Gastroenterol. 17, 534-539.

Choi, B. M., Kim, H. R., Yun, H. R., Choi, S. H., Cho, Y. B., Kim, H. C., Yun, S. H., Lee, W.Y., Chun, H. K. (2011) Treatment outcomes of anorectal melanoma. J. Korean Soc. Coloproctol. 27, 27-30.

Chute, D. J., Cousar, J. B., Mills, S. E. (2006) Anorectal malignant melanoma morphologic and immunohistochemical features. Am. J. Clin. Pathol. 126, 93-100.

Damodaran, O., Morgan, A., Meldelsohn, G. (2008) Primary malignant melanoma in the rectum: an uncommon cancer. N. Z. Med.J. 121, 66-68.

Falch, C., Stojadinovic, A., Hann-von-Weyhern, C., Protic, M., Nissan, A., Faries, M. B., Daumer, M., Bilchik, A. J., Itzhak, A., Brücher, B. L. (2013) Anorectal malignant melanoma: extensive 45-year review and proposal for a novel staging classification. J. Am. Coll. Surg. 217, 324-335.

Goldman, S., Glimelius, B., Pahlman, L. (1990) Anorectal malignant melanoma in Sweden. Report of 49 patients. Dis. Colon Rectum 33, 874-877.

Ishizone, S., Koide, N., Karasawa, F., Akita, N., Muranaka, F., Uhara, H., Miyagawa, S. (2008) Surgical treatment for anorectal malignant melanoma: report of five cases and review of 79 Japanese cases. Int. J. Colorectal Dis. 23, 1257-1262.

Kelly, P., Zagars, G. K., Cormier, J. N., Ross, M. I., Guadagnolo, B. A. (2011) Sphincter-sparing local excision and hypofractionated radiation therapy for anorectal melanoma: a 20-year experience. Cancer 117, 4747-4755.

Malik, A., Hull, T. L., Milsom, J. (2002) Long-term survivor of anorectal melanoma: report of a case. Dis. Colon Rectum 45, 1412-1415.

Ojima, Y., Nakatsuka, H., Haneji, H., Kurihara, T., Sadamoto, S., Ohmoto, T., Katayama, N., Tanijama, K. (1999) Primary anorectal malignant melanoma: report of a case. Surg. Today 29, 170-173.

Perez, D. R., Trakarnsanga, A., Shia, J., Nash, G. M., Temple, L. K., Paty, P. B., Guillem, J. G., Garcia-Aguilar, J., Bello, D., Ariyan, C., Carvajal, R. D., Weiser, M. R. (2013) Locoregional lymphadenectomy in the surgical management of anorectal melanoma. Ann. Surg. Oncol. 20, 2339-2344.

Pessaux, P., Pocard, M., Elias, D., Duvillard, P., Avril, M. F., Zimmerman, P., Lasser, P. (2004) Surgical management of primary anorectal melanoma. Br. J. Surg. 91, 1183-1187.

Ramalingam, G., Gan, E.Y., Kut-Sing,W. (2009) Laparoscopic abdominoperineal resection for anorectal melanoma: a case report and review of the literature. Surg. Laparosc. Endosc. Percutan. Tech. 19, 149-151. 
106) Prague Medical Report / Vol. 119 (2018) No. 2-3, p. 97-106

Reid, A., Dettrick, A., Oakenful, C., Lambrianides, A. L. (2011) Primary rectal melanoma. J. Surg. Case Rep. 2011(11), 2.

Row, D., Weiser, M. R. (2009) Anorectal melanoma. Clin. Colon Rectal Surg. 22, 120-126.

Sielezneff, I., Boutboul, R., Thomas, P., Henric, A., Denis, O. (1993) Primary anorectal malignant melanomas. 2 cases. Presse Med. 22, 1999-2001. (in French)

Stefanou, A., Nalamati, S. P. (2011) Anorectal melanoma. Clin. Colon Rectal Surg. 24, 171-176.

Thibault, C., Sagar, P., Nivatvongs, S., Ilstrup, D. M., Wolff, B. G. (1997) Anorectal melanoma - an incurable disease? Dis. Colon Rectum 40, 661-668.

Tien, H.Y., Mc Masters, K. M., Edwards, M. J., Chao, C. (2002) Sentinel lymph node metastasis in anal melanoma: a case report. Int. J. Gastrointest. Cancer 32, 53-56.

Wanebo, H. J., Woodruff, J. M., Farr, G. H., Quan, S. H. (1981) Anorectal melanoma. Cancer 47, 1891-1900.

Weinstock, M. (1993) Epidemiology and prognosis of anorectal melanoma. Gastroenterology 104, 174-178.

Yap, L. B., Neary, P. (2004) A comparison of wide local excision with abdominoperineal resection in anorectal melanoma. Melanoma Res. 14, 147-150.

Yeh, J. J., Shia, J., Hwu, W. J., Busam, K. J., Paty, P. B., Guillem, J. G., Coit, D. G., Wong, W. D., Weiser, M. R. (2006). The role of abdominoperineal resection as surgical therapy for anorectal melanoma. Ann. Surg. 244, 1012-1017. 